a shear of $14^{\prime}$ in the $a-c$ plane being observed at $-16^{\circ}$ C. $\left(\beta=89^{\circ} 46^{\prime}\right)$. Associated with this change there is an expansion of the $a$ parameter and a slight contraction of $c$, such that these two parameters become equal, and the polar direction turns through $45^{\circ}$ into the $[011]$ or $[101]$ direction. A further transition occurs near $-70^{\circ} \mathrm{C}$., below which tem. perature the symmetry appears to be rhombohedral with the polar axis in the former [111] direction.

$$
\begin{aligned}
& \text { H. F. KAY } \\
& \text { H. J. WELLARD } \\
& \text { P. VOUSDEN }
\end{aligned}
$$

H. H. Wills Physical Laboratory,

University of Bristol. Oct. 20.

'Megaw, Proc. Roy. Soc., A, 189, 261 (1947).

${ }^{2}$ Discussion of Flectronics Group of Institute of Physics, Nature, $162,245(1948)$.

${ }^{s}$ Hulm, Nature, 160, 127 (1947).

\section{A New Application of White-Light Superposition Fringes}

WHITE-LIGHT superposition fringes have found a number of applications in the past. They are of two kinds-fringes of equal inclination and of equal thickness. Extensive use of the former has been made, first in Brewster fringes, later in the Jamin interferometer ; then in 1898 Fabry and Buisson first used multiple. beams, ultimately adopting the fringes for their classical evaluation of the metre. A more recent application has been made by Sears and Barrell in determining the refractive index of air.

Comparable use of white-light superposition fringes of equal thickness has not, however, been made; apart from a few experiments described by Fabry in his book "Les Applications des Interférences Lumineuses" (1924), there is little to record. It is the purpose of this note to describe a simple applica. tion which reveals qualitative information with high precision.

It has already been demonstrated by interferometry that many varieties of muscovite mica cleave surprisingly true over large areas. A randomly selected sheet of muscovite was silvered on both sides. It was then cut into two with scissors and the two pieces held superposed on each other. The combination was placed in the parallel beam of a standard Fizeau fringe set-up, but with white light. Thus an image of a 'Pointolite' is thrown on to an aperture which is at the principal focus of a lens, and the emergent parallel beam of white light directed at normal incidence on to the mica combination. A lens follows the mica, and the eye is placed at the lens focus. The appearance is striking. Following recognized principles, it is clear that localized whitelight superposition fringes of equal thickness will form. If the two pieces of mica consisted of pieces of strictly constant thickness, then the field of view would consist merely of a uniform tint of illumination. But, in fact, there are cleavage steps on mica surfaces and, as is already known, these usually occur in simple multiples of the crystal lattice spacing $(20 \mathrm{~A}$. and may, in fact, be only a single lattice high. It is clear that the superposition principle leads effectively to the production of thin-film interference over pathdifferences representing the heights of the cleavage steps. The result is that the field of view shows brilliantly coloured areas, each of uniform tint, each being delineated by the boundary of cleavage lines. Thus, in a highly sensitive (but only qualitative) manner, the structure of the mica sheet is revealed.
For not only do height changes lead to colour variations, but also inclusions and variations of refractive index have a like effect.

It remains to ask whether this rather restricted new technique has any advantages over the monochromatic multiple-beam method already in use for thin films. The answer is, yes. In two respects the new method is a decided improvement on the old. First, when using monochromatic light, the very sharpness of the fringe distribution is such that cleavage steps may easily be missed if the trans. mission region corresponds to a thickness well off the intensity maximum; secondly, it can be readily demonstrated by simple calculation that the fringewidth in the combination system is $1 / \sqrt{ } 2$ that in the single system. There is, therefore, a 40 per cent increase in sensitivity.

It is to be emphasized that the interference colours are determined primarily by the individual stepheights and not by the thickness of the mica sheet. With very strict collimation, it should certainly be possible to apply the technique to thick slabs. This being so, equal slabs of optical material can be exactly matched and accurately surfaced, for ex. ample, with the dividing plate and compensating plate in a Michelson interferometer, or the plates for a reflexion echelon, etc. Other applications, with both thin and thick interference gaps, come readily to mind.

\section{Royal Holloway College,}

Englefield Green, Surrey. Oct. 29 .

\section{Influence of a Magnetic Field on the Size- Variation of Electrical Conductivity}

Miss I. STONE ${ }^{1}$, in 1898 , appears to have been one of the first to make a study of the anomalous increase in specific resistivity of very thin metal films as compared with specimens in bulk, although the phenomenon was already known. Films of silver as thin as $\sim 1 \times 10^{-6} \mathrm{~cm}$. deposited by the 'Rochelle salt process' were used by her. Longden" extended this work to evaporated films of platinum, and since that time a considerable amount of work has been carried out on such thin films (for example, bibliography in ref. 3). J. J. Thomson first proposed that the physical limitation of conductor size would produce a shortening of the electron mean free path, with a consequent reduction of effective conductivity, and derived a theoretical expression based on an assumption of a constant mean free path in the bulk metal.

More recently, the accessibility of very low temperatures has enabled the effect to be observed in specimens of considerably larger size $(\sim 10-100 \mu)$, with the resulting elimination of a number of sub. sidiary effects which arise in very thin films. In particular, Andrew ${ }^{5}$ has studied wires of pure mercury and wires and foils of pure tin in the region $1-4^{\circ} \mathrm{K}$. One difficulty, emphasized by Andrew, in the interpretation of the data is that of relating the measured conductivity to that of an equivalent bulk sample, since strain and impurity--both productive of high residual resistance at low temperatures-may be expected to play a significant part in finer specimens.

Modern theories of metallic conduction as applied to the phenomenon ${ }^{6}$ require an assumption of the degree of 'freeness' of the conduction electrons. It 